\title{
Quality of Life in Patients with Chronic Thyroid Eye Disease in the United States
}

\author{
Kimberly P. Cockerham • Lissa Padnick-Silver • Noel Stuertz • \\ Megan Francis-Sedlak · Robert J. Holt
}

Received: July 12, 2021 / Accepted: August 9, 2021 / Published online: September 3, 2021

(C) The Author(s) 2021, corrected publication 2022

\section{ABSTRACT}

Introduction: Thyroid eye disease (TED) is an autoimmune condition producing ocular pain, dysmotility, and ocular structure and function changes. As disease activity changes, redness, swelling, and pain can improve, but eye comfort, appearance, and motility alterations often persist. There are limited data on chronic TED

Supplementary Information The online version contains supplementary material available at https:// doi.org/10.1007/s40123-021-00385-8.

K. P. Cockerham

Central Valley Eye Medical Group, 36 W Yokuts

Avenue, Suite 2, Stockton, CA 95207, USA

K. P. Cockerham

Department of Ophthalmology, Stanford School of Medicine, 2454 Watson Court, Palo Alto, CA 94303, USA

e-mail: cockerhammd@gmail.com

L. Padnick-Silver · N. Stuertz · M. Francis-Sedlak ·

R. J. Holt $(\bowtie)$

Horizon Therapeutics plc, 1 Horizon Way, Deerfield, IL 60015, USA

e-mail: rholt@horizontherapeutics.com

L. Padnick-Silver

e-mail: lsilver@horizontherapeutics.com

N. Stuertz

e-mail: nstuertz@horizontherapeutics.com

M. Francis-Sedlak

e-mail: mfrancis-sedlak@horizontherapeutics.com patient-reported outcomes. This study examined chronic US TED patient-reported symptoms and quality of life (QOL).

Methods: Existing data from an online survey regarding chronic TED signs/symptoms and patient QOL were retrospectively examined. The Graves' Ophthalmopathy QOL instrument (GO-QOL; 0-100, 100 = highest QOL) evaluated overall, appearance, and vision-related QOL. Influencing factors were examined by stratifying patients into low (overall QOL $\leq 50$ ), moderate $(>50$ and $<75)$, and high $(\geq 75)$ QOL categories.

Results: One hundred patients (47 women, 81 Caucasian, $45.2 \pm 7.6$ years) were included. The duration of inactive TED was $3.0 \pm 4.6$ years and total duration of TED was $5.8 \pm 5.9$ years. Patients reported an average of 20 doctor visits/ year and high prevalence of anxiety (34\%) and depression (28\%). Prior TED treatments for the polled population included systemic corticosteroids during active TED (25\%), orbital radiation (5\%), and surgery (25\%). The overall GOQOL score was $60.5 \pm 21.8$ (vision-related: $58.6 \pm 24.0$, appearance-related: $62.3 \pm 25.1$ ). Patients with low QOL more frequently reported hypothyroidism, anxiety, and a larger number of chronic TED signs/symptoms (average: 4.2). Compared to high QOL patients, low QOL patients had more pain (39\% vs. $13 \%)$, blurry vision ( $30 \%$ vs. $17 \%)$, and diplopia ( $27 \%$ vs. $3 \%$, all $p \leq 0.025$ ). Additionally, the low QOL group more often had TED-specific 
surgical history ( $45 \%$ vs. $10 \%, p=0.002$ ), more often reported disability/unemployment (21\% vs. $3 \%, p=0.055$ ), and had a higher number of doctor visits (40 vs. 5 visits/person/year, $p<0.001$ ).

Conclusion: TED severely impacts patient QOL, despite becoming stable and chronic. Patients reported vision and appearance impairment and psychosocial impact long after acute TED had subsided.

\section{PLAIN LANGUAGE SUMMARY}

Thyroid eye disease (TED) occurs when loss of immune tolerance results in orbital and retroorbital inflammation. Fat and muscle tissue can swell severely, causing debilitating symptoms, including pain around/behind the eyes, eye movement abnormalities, bulging eyes (proptosis), and double vision (diplopia), manifesting in appearance and vision quality of life (QOL) changes. Some improvement can occur as inflammation quiets and TED becomes chronic/ inactive. However, appearance and visual changes often remain due to persistent proptosis and eye muscle and eyelid changes. This study examined TED symptoms and QOL in 100 chronic TED patients. They answered questions about symptoms, how TED affected them, and their medical care. The average duration of TED was 6 years ( 3 years inactive), patients had an average of 20 TED-related doctor visits/year, and nearly one-half $(42 \%)$ of patients reported having anxiety and/or depression. Prior TED treatments included steroids (25\% when TED-related inflammation was present), orbital radiation (5\%), and surgery (25\%). Disease-specific QOL scores (average score: 60.5 of 100) indicated that these chronic patients reported similar QOL impact as those with moderate-to-severe, active disease. Compared with the least impacted group, the most impacted patients reported higher rates of hypothyroidism (18\% vs. 0\%), anxiety ( $48 \%$ vs. $17 \%)$, disability/unemployment $(21 \%$ vs.3\%), number of doctor visits (40 vs. 5 visits/person/year), pain (39\% vs. $13 \%)$, blurry vision ( $30 \%$ vs. $17 \%)$, diplopia ( $27 \%$ vs. $3 \%)$, and surgical treatment for TED ( $45 \%$ vs.
10\%). This study demonstrates that QOL continues to be severely impacted by TED long after TED-related inflammation has quieted.

Keywords: Thyroid eye disease; Inactive; Chronic; Quality of life; Clinical presentation

\section{Key Summary Points}

\section{Why carry out this study?}

The literature on chronic thyroid eye disease (TED) is sparse, particularly that pertaining to long-term disease, but a better understanding is crucial as medical therapies for all stages of TED emerge.

This study examined QOL and TED signs and symptoms in 100 chronic TED patients in the United States.

\section{What was learned from the study?}

Patients had long-term TED (average duration: 6 years [3 years chronic, inactive TED]), but low QOL (average score: 61 out of 100), frequent mental health issues (42\%), and high healthcare utilization (average: 20 visits/patient in the year prior to survey).

Patient QOL continued to be severely impacted by persistent TED signs and symptoms long after TED-related inflammation had quieted.

\section{INTRODUCTION}

Thyroid eye disease (TED) is an autoimmune disease that causes inflammation and expansion of the muscles and fat in the orbit. These structural changes result in pain, ocular dysmotility, blurred vision, and appearance changes that result in a low quality of life (QOL). Acute, active disease generally presents with ocular redness, conjunctival swelling, eyelid redness and swelling, and/or pain behind or around the eyes. Retro-orbital fat tissue and 
extraocular muscles can enlarge markedly, leading to proptosis, diplopia, and/or compressive optic neuropathy which may compromise vision. Eyelid retraction can result in an incomplete blink and/or alterations in the tear film, causing tearing, irritation, and blurred vision. Even though TED signs and symptoms can improve as inflammation subsides, many persist over the long term [1] and continue to negatively impact QOL. More specifically, patients' ability to work $[2,3]$, perform everyday tasks $[4,5]$, and maintain social relationships $[5,6]$ have been shown to chronically suffer, along with mental health $[5,7,8]$. Many patients only have one initial inflammatory episode, but an estimated $16 \%$ have an inflammatory flare after at least 6 months of stability [9], and 5\% have a flare after at least 5 years of stability [10].

A recent study examined QOL in active, moderate-to-severe TED patients in the United States (US), showing a large QOL impact and mental health burden [11]. One older study in the US $(n=120)$ showed that TED patients continued to suffer from ocular discomfort and appearance dissatisfaction years after their initial TED diagnosis (mean follow-up time: 9.8 years) [12]. Additionally, Dutch TED patients $(n=154)$ who had been treated with radiotherapy or prednisone treatment continued to suffer over the long term (median followup time: 11.7 years) from proptosis (60\%), diplopia (51\%), and impaired visual acuity (worse than 20/25, 28\%) [5]. As a result, both physical and mental function were markedly impaired [5]. Further, QOL was previously evaluated in two placebo-controlled trials in 171 randomized moderate-to-severe active TED patients with recent ( $\leq 9$ months) symptom onset. Baseline QOL was measured in these patients with a TED-specific instrument (Graves' Ophthalmopathy Quality of Life questionnaire [GO-QOL]) and demonstrated a low mean QOL score (61 out of 100) [13]. However, the longterm QOL impact of chronic TED on US patients has not been well reported. Additionally, information regarding which TED signs and symptoms persist over the long term and how these impact patient QOL is sparse. Given the ongoing development of new therapies for all phases of TED, a better understanding of chronic TED and how it impacts patient QOL is of the utmost importance. The current survey aimed to better understand the US TED population, particularly chronic TED sign and symptom persistence that interfered with tasks of daily life and impacted overall patient QOL. Potential factors that influenced QOL were also investigated.

\section{METHODS}

We analyzed existing, de-identified patient survey data. Therefore, this study was assigned exempt status by the Western Institutional Review Board (IRB; Puyallup, WA). All study conduct adhered to the tenets of the Declaration of Helsinki. Patients who completed the survey were paid $\$ 85$ as reimbursement for their time.

One hundred patients with inactive/chronic TED completed an online survey in January 2020. Patients with all levels of TED severity were included. A third party (Instar, New York, NY) designed the survey in collaboration with the sponsor (Horizon Therapeutics, Deerfield, IL), administered the survey, and collated data in a de-identified manner before providing it to the authors for analysis. After confirming they had a formal diagnosis of "thyroid eye disease (TED) also called Graves ophthalmopathy, thyroid-associated ophthalmopathy, and Graves orbitopathy," patients were asked, "Is your thyroid eye disease (TED) currently active or inactive?" Patients were provided brief descriptions of both active and inactive TED for reference (Supplemental Table 1). Participants were also asked, "What phrase did the healthcare professional use when they told you that your thyroid eye disease (TED) was inactive?" The following key words/phrases were looked for as confirmation of TED inactivity: quiet, stable, inactive/no longer active, improving/overall better, dormant, less irritated/inflamed, not a lot of redness, and no longer changing.

The survey included items pertaining to demographic information, smoking status, comorbidities, age at TED diagnosis and at time of survey, TED treatment history, and thyroid 
Table 1 Characteristics of inactive, chronic thyroid eye disease survey respondents

\begin{tabular}{|c|c|}
\hline Patient characteristics & $N=100$ \\
\hline \multicolumn{2}{|l|}{ Age at survey, years } \\
\hline Mean $\pm S D$ & $45.2 \pm 7.6$ \\
\hline Median (range) & $45(23-64)$ \\
\hline \multicolumn{2}{|l|}{ Age at TED diagnosis, years } \\
\hline Mean \pm SD & $39.5 \pm 9.7$ \\
\hline Median (range) & $40(17-60)$ \\
\hline Female, $n(\%)$ & $47(47 \%)$ \\
\hline \multicolumn{2}{|l|}{ Patient ethnicity, $n$ (\%) } \\
\hline Caucasian & $81(81 \%)$ \\
\hline African-American & $11(11 \%)$ \\
\hline Asian & $4(4 \%)$ \\
\hline Native American & $2(2 \%)$ \\
\hline Other (Hispanic, biracial) & $2(2 \%)$ \\
\hline \multicolumn{2}{|l|}{ Smoking status, $n(\%)$} \\
\hline Current smoker & $13(13 \%)$ \\
\hline Former smoker & $26(26 \%)$ \\
\hline Never-smoked & $61(61 \%)$ \\
\hline \multicolumn{2}{|l|}{ Most common comorbidities, $n(\%)$} \\
\hline Non-thyroid autoimmune disease & $24(24 \%)$ \\
\hline Type 1 diabetes & $5(5 \%)$ \\
\hline Inflammatory bowel disease & $5(5 \%)$ \\
\hline Rheumatoid arthritis & $5(5 \%)$ \\
\hline Lupus & $4(4 \%)$ \\
\hline Psoriasis & $3(3 \%)$ \\
\hline Psoriatic arthritis & $3(3 \%)$ \\
\hline Autoimmune blood disorder & $1(1 \%)$ \\
\hline Unspecified autoimmune disease & $1(1 \%)$ \\
\hline Myasthenia gravis & $1(1 \%)$ \\
\hline Hypertension & $22(22 \%)$ \\
\hline Overweight/obese & $16(16 \%)$ \\
\hline
\end{tabular}

Table 1 continued

\begin{tabular}{ll}
\hline Patient characteristics & $\boldsymbol{N}=\mathbf{1 0 0}$ \\
\hline TED duration, mean \pm SD, years & \\
Active/acute disease & $2.7 \pm 2.5$ \\
Inactive/chronic disease & $3.0 \pm 4.6$ \\
Total TED duration & $5.8 \pm 5.9$ \\
\hline
\end{tabular}

$\mathrm{SD}$, standard deviation; TED, thyroid eye disease

levels (high, normal, or low). Treatment history items included both medical (i.e., over-thecounter pain relievers [acetaminophen, NSAIDs], glucocorticoids [topical, oral, intravenous], and biologics [rituximab, tocilizumab]) and surgical/procedural (i.e., orbital decompression, strabismus, eyelid, cosmetic, orbital radiation) treatments. Patient characteristics and TED parameters were inquired about both at the time of diagnosis and after patients were told their TED had become inactive (applicable survey items listed in Supplemental Table 2). QOL in patients with chronic TED was measured using the English version [14] of the Graves' Ophthalmopathy Quality of Life (GOQOL) instrument [15]. GO-QOL scores range from 0 (worst) to 100 (best), with a score of 90-100 considered normal. Overall QOL scores, along with appearance-related and visual function QOL sub-scores, were examined. Factors that potentially influenced QOL were examined by stratifying patients by overall GO-QOL score into the following terciles: low QOL (less than or equal to 50), middle QOL (between 50 and 75 ), and high QOL (greater than or equal to 75 ).

Algorithms were used to verify that data were from actual patients, identify verbatim responses, and monitor survey completion time for responses that were too rapid to be accurate or real. Incomplete surveys and those suspected of containing low-quality or imposter responses were excluded from analyses. Data are presented as mean \pm standard deviation or $n(\%)$ as appropriate. Differences between groups were examined using unpaired, two-tailed Student's $t$-tests for continuous parameters and two-tailed Fisher's exact tests for categorical parameters. Statistical significance was defined as $p<0.05$. 

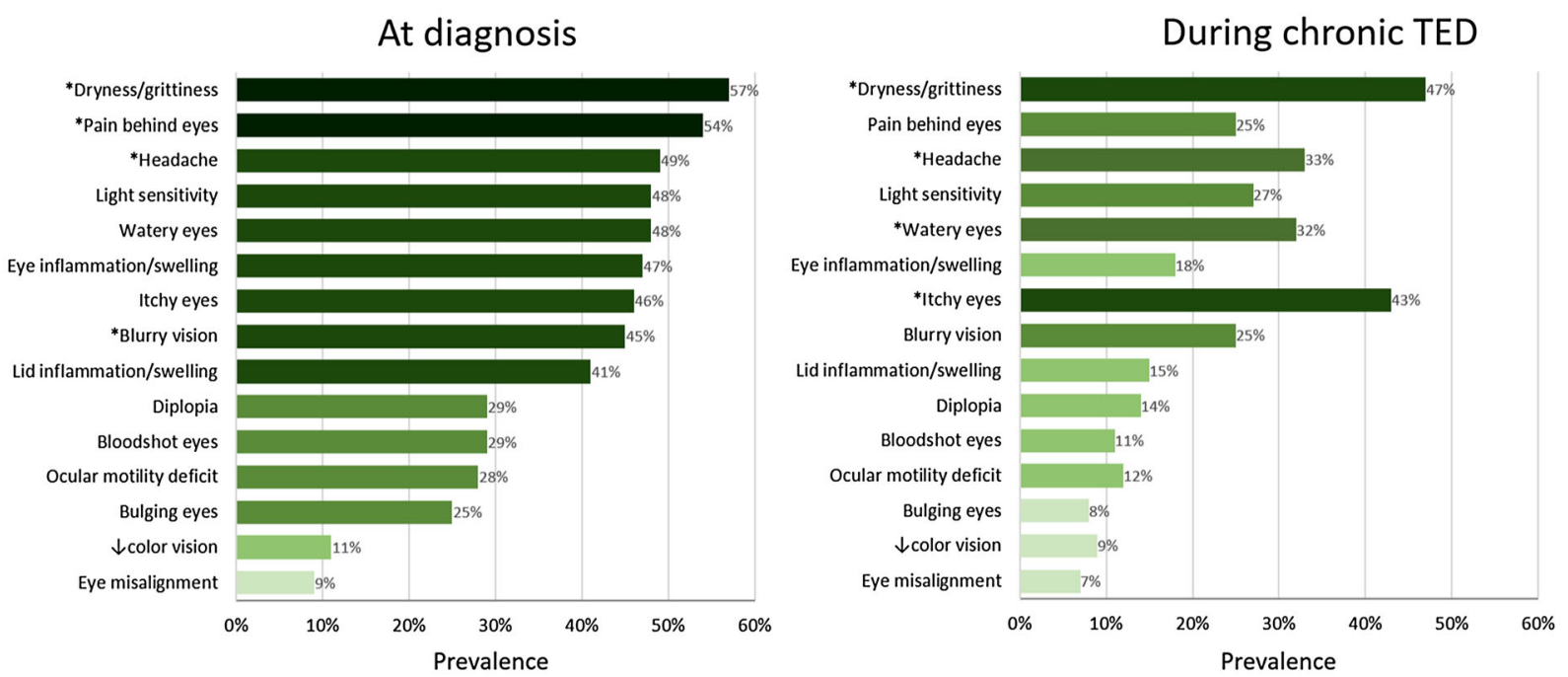

Fig. 1 Prevalence of patient-reported thyroid eye disease (TED) symptoms at diagnosis and after they had been told their TED had become inactive or chronic. *Indicates most bothersome symptom in $\geq 10 \%$ of patients

\section{RESULTS}

A total of 4950 patients in an existing research database were emailed an invitation to complete the survey. All patients had been profiled as having a thyroid condition, Graves' disease, or TED to maximize the chances of reaching a TED patient (1497 [30\%] previously reported TED or Graves' disease, 3453 [70\%] previously reported a thyroid condition). Of these, 773 $(16 \%)$ clicked on the survey link to indicate their interest, with 206 inactive TED patients (27\%) ultimately identified (567 patients did not have inactive TED). Of these 206 inactive TED patients, 100 were included in this analysis (67 did not complete the survey, 39 surveys removed for quality issues). Participants were geographically well distributed across the United States, with 35 states represented (Supplemental Figure).

\section{Patient Characteristics}

The 100 included patients had an average age of $45.2 \pm 7.6$ years at the time of survey (Table 1 ). Approximately half of patients were female $(47 \%)$ and most were Caucasian $(81 \%)$. The majority of patients were non-smokers $(87 \%)$ at the time of survey, and comorbidity prevalence was fairly low, with non-thyroid autoimmune disorders (24\%) and hypertension (22\%) most commonly reported. At the time of survey, thyroid levels were self-reported as "normal" in $63 \%$ of patients, "too high" in $23 \%$ of patients, and "too low" in 14\% of patients. Male and female patients were similar in terms of age $(43.8 \pm 6.8$ and $46.8 \pm 8.2$ years), but male patients reported lower rates of obesity ( $8 \%$ vs. $26 \%$ ), anxiety (23\% vs. $47 \%)$, cancer ( $0 \%$ vs. $11 \%)$, chronic fatigue syndrome ( $0 \%$ vs. $15 \%)$, fibromyalgia ( $2 \%$ vs. $17 \%)$, osteoarthritis $(0 \%$ vs. $17 \%$ ), and ulcerative colitis/Crohn's/inflammatory bowel disease $(0 \%$ vs. $11 \%$; all $p \leq 0.027$ ).

Average TED duration was reported as $5.8 \pm 5.9$ years, with $2.7 \pm 2.5$ years in the active, progressive phase and $3.0 \pm 4.6$ years in the inactive, chronic phase (Table 1). Most patients reported receiving their thyroid diagnosis before $(45 \%)$ or at the same time $(40 \%)$ as their TED diagnosis, with $45 \%$ receiving the two diagnoses within a 6-month period and 67\% within 18 months. At TED diagnosis, the most commonly reported symptoms included ocular dryness/grittiness $(57 \%)$, pain behind the eyes (54\%), headache (49\%), light sensitivity (48\%), watery eyes $(48 \%)$, eye inflammation/swelling 
Table 2 Characteristics of patients in quality-of-life terciles, as measured using the thyroid eye disease-specific GO-QOL instrument

\begin{tabular}{|c|c|c|c|c|}
\hline Overall GO-QOL score & $\begin{array}{l}\text { Low QOL } \\
\leq 50 \\
(n=33)\end{array}$ & $\begin{array}{l}\text { Middle QOL } \\
>50 \text { and }<75 \\
(n=37)\end{array}$ & $\begin{array}{l}\text { High QOL } \\
\geq 75 \\
(n=30)\end{array}$ & $\begin{array}{l}p \text {-value } \\
\text { (low vs. high) }\end{array}$ \\
\hline Female, $n(\%)$ & $15(45 \%)$ & $20(54 \%)$ & $12(40 \%)$ & 0.800 \\
\hline Caucasian, $n(\%)$ & $23(70 \%)$ & $31(84 \%)$ & $27(90 \%)$ & 0.064 \\
\hline Patient age, mean $\pm S D$, years & $43.8 \pm 6.6$ & $46.6 \pm 8.6$ & $44.9 \pm 7.2$ & 0.528 \\
\hline \multicolumn{5}{|l|}{$\mathrm{TED}$ duration, mean $\pm \mathrm{SD}$, years } \\
\hline Total TED duration & $7.0 \pm 6.9$ & $4.6 \pm 4.8$ & $5.8 \pm 6.0$ & 0.473 \\
\hline Active/acute TED duration & $2.6 \pm 2.3$ & $2.7 \pm 2.9$ & $2.8 \pm 2.2$ & 0.729 \\
\hline Inactive/chronic TED duration & $4.4 \pm 6.0$ & $1.9 \pm 3.0$ & $3.0 \pm 4.3$ & 0.299 \\
\hline \multicolumn{5}{|l|}{ Patient-reported thyroid hormone levels, $n$ (\%) } \\
\hline Normal & $18(55 \%)$ & $18(49 \%)$ & $27(90 \%)$ & 0.002 \\
\hline Too low & $6(18 \%)$ & $8(22 \%)$ & $0(0 \%)$ & 0.025 \\
\hline Too high & $9(27 \%)$ & $11(30 \%)$ & $3(10 \%)$ & 0.112 \\
\hline Disabled/unemployed, $n$ (\%) & $7(21 \%)$ & $6(16 \%)$ & $1(3 \%)$ & 0.055 \\
\hline Mental health issue ${ }^{\mathrm{a}}, n(\%)$ & $18(55 \%)$ & $16(43 \%)$ & $8(27 \%)$ & 0.040 \\
\hline Anxiety & $16(48 \%)$ & $13(35 \%)$ & $5(17 \%)$ & 0.009 \\
\hline Depression & $12(36 \%)$ & $10(27 \%)$ & $6(20 \%)$ & 0.174 \\
\hline \multicolumn{5}{|l|}{ Number of doctor visits/person/year ${ }^{b}$} \\
\hline Mean $\pm S D$ & $40.2 \pm 46.3$ & $13.5 \pm 15.2$ & $4.8 \pm 4.9$ & $<0.001$ \\
\hline Median (range) & $14(2-160)$ & $7(1-60)$ & $4(1-24)$ & -- \\
\hline Patients with a history of TED-related procedure ${ }^{c}, n(\%)$ & $15(45 \%)$ & $9(24 \%)$ & $3(10 \%)$ & 0.002 \\
\hline Number of chronic TED signs/symptoms, mean \pm SD & $4.2 \pm 2.5$ & $3.5 \pm 2.0$ & $2.2 \pm 1.2$ & $<0.001$ \\
\hline Pain behind the eyes, $n$ (\%) & $13(39 \%)$ & $8(22 \%)$ & $4(13 \%)$ & 0.025 \\
\hline Light sensitivity, $n(\%)$ & $12(36 \%)$ & $11(30 \%)$ & $4(13 \%)$ & 0.046 \\
\hline Blurry vision, $n$ (\%) & $10(30 \%)$ & $10(27 \%)$ & $5(17 \%)$ & 0.024 \\
\hline Diplopia, $n(\%)$ & $9(27 \%)$ & $4(11 \%)$ & $1(3 \%)$ & 0.014 \\
\hline
\end{tabular}

Quality of life measured at the time of survey during chronic TED. Two-tailed $p$-values calculated with unpaired $t$-tests for continuous variables and Fisher's exact tests for categorical variables

a Includes patients who reported anxiety and/or depression

b Reported for the 12 months prior to survey

c Includes orbital decompression, strabismus surgery, eyelid surgery, cosmetic surgery, and orbital radiation. QOL, quality of life 
(47\%), itchy eyes (46\%), blurry vision (45\%), and lid inflammation/swelling (41\%, Fig. 1). Diplopia was reported in $29 \%$ of patients at TED diagnosis. TED symptoms were reported at a similar rate in men and women, with the exception of watery eyes $[36 \%$ vs. $62 \%$, $p=0.016]$, dryness/grittiness $[47 \%$ vs. $68 \%$, $p=0.044]$, and light sensitivity [36\% vs. $62 \%$, $p=0.016]$, which were all reported less often in men.

Symptoms that most commonly persisted into chronic disease included dryness/grittiness (47\%), itchy eyes (43\%), headache (33\%), watery eyes (32\%), light sensitivity (27\%), blurry vision $(25 \%)$, and pain behind the eyes (25\%, Fig. 1). Diplopia was reported during chronic TED in $14 \%$ of patients. Ocular dryness/ grittiness, headache, watery eyes, and itchy eyes were noted as the most bothersome symptom during chronic TED ( $\geq 10 \%$ of patients). Of note, headache and itching are not typical symptoms attributed to either acute or chronic TED. Male and female patients experienced similar symptoms during chronic TED, except that women more often reported light sensitivity $(38 \%$ vs. $17 \%, p=0.024)$.

\section{Effect of Thyroid Eye Disease on Quality of Life and Mental Health}

Patient QOL was markedly impaired by TED at the time of survey, as indicated by an overall GO-QOL score of $60.5 \pm 21.8$, an appearance sub-score of $62.3 \pm 25.1$, and a visual function sub-score of $58.6 \pm 24.0$. Of note, $70 \%$ of patients had an overall GO-QOL score $<75$, and $33 \%$ of patients had an overall score of $\leq 50$. Additionally, $42 \%$ of patients reported having anxiety and/or depression (34\% reported anxiety, 28\% reported depression), more than double the prevalence of mental health issues in the general US adult population (National Institutes of Health 2019 statistic for prevalence of "any mental health issue": $20.6 \%$ [16]). Further, patients sought medical care often for their TED, with an average of $19.7 \pm 31.7$ TED-related doctor visits/patient reported in the year prior to survey. Endocrinologists and ophthalmologists were most often seen, followed by optometrists and primary care physicians. Fourteen percent of patients reported being disabled/unemployed at the time of survey.

\section{Factors that Influenced Quality of Life}

Patients were divided into terciles of low, middle, and high QOL groups to examine factors that potentially influenced QOL. Characteristics of each QOL group are summarized in Table 2. Briefly, patient demographics were similar, but mental health issues were more prevalent in the low versus high QOL tercile (55\% vs. $27 \%$, $p=0.040$ ). Additionally, more patients reported being disabled or unemployed in the low QOL group ( $21 \%$ vs. $3 \%, p=0.055)$. TED-related differences between the high and low QOL terciles were identified and included the number of TED-related doctor visits, a history of TED surgery (Fig. 2b), and the number of TED signs and symptoms reported during chronic TED (Fig. 2a). More specifically, pain behind the eyes (39\% vs. $13 \%, p=0.025)$, light sensitivity $(36 \%$ vs. $13 \%, p=0.046)$, blurry vision $(30 \%$ vs. $17 \%$, $p=0.024)$, and diplopia ( $27 \%$ vs. $3 \%, p=0.014)$ were present significantly more often in the low versus high QOL tercile. Additionally, more patients in the high versus low QOL group had normal thyroid hormone levels (90\% vs. 55\%, $p=0.002$ ), with low levels negatively impacting QOL (Table 2). Further, patients who underwent one or more TED-related procedures (including orbital decompression, strabismus surgery, eyelid surgery, cosmetic surgery, and/or orbital radiation) had significantly lower GO-QOL scores at the time of survey than patients who had not undergone surgery (overall: $51.8 \pm 16.8$ vs. $63.7 \pm 22.6$, visual function: $50.8 \pm 20.1$ vs. $61.5 \pm 24.9, \quad$ appearance: $\quad 53.2 \pm 19.4$ vs. $65.7 \pm 26.3$; all $p \leq 0.031$ ). Of note, more male patients had undergone orbital decompression ( $21 \%$ vs. $6 \%, p=0.019$ ), but overall GO-QOL score was nearly identical at data collection (male: $\quad 60.7 \pm 22.2, \quad$ female: $\quad 60.1 \pm 21.5$; $p=0.893)$. 


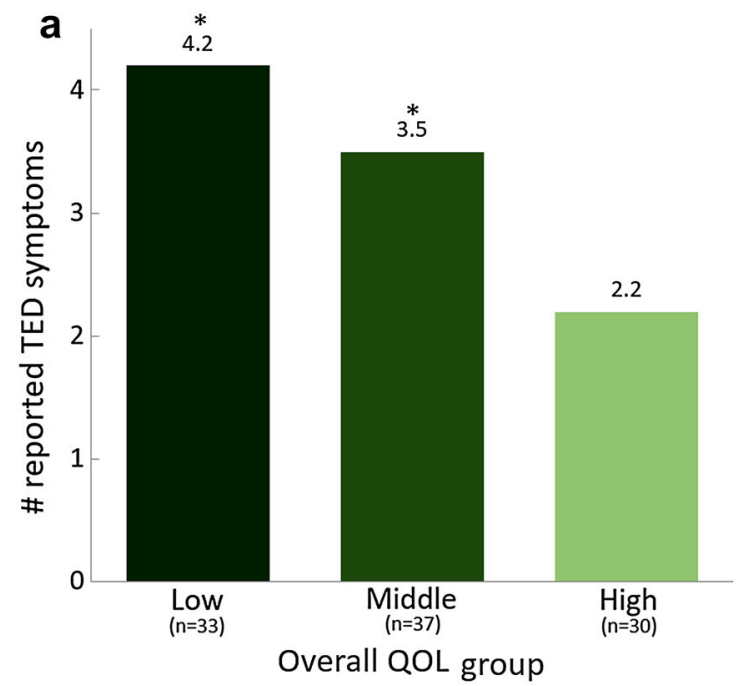

Fig. 2 a Mean number of TED signs and symptoms experienced by patients during the chronic phase of TED. b Proportion of patients with a TED-specific surgical history in the low, middle, and high tercile QOL groups. The number of patients who underwent at least one TEDrelated procedure was significantly higher in the low QOL tercile than in the high QOL tercile $(45 \%$ vs. $10 \%$ [includes orbital radiation], $p=0.002)$. Percentages represent the proportion of patients within each QOL tercile

\section{Systemic Glucocorticoid Use During Active Thyroid Eye Disease}

A total of 25 patients (25\%) had been treated with oral and/or intravenous (IV) glucocorticoids during active TED (21 patients oral only, 1 patient IV only, 3 patients both oral and IV). Patient characteristics were similar between patients with and without prior systemic glucocorticoid use. At the time of survey, there was no patient-reported difference in overall, visionrelated, or appearance-related QOL between patients previously treated and not treated with systemic glucocorticoids during active, progressive TED (Fig. 3). However, patients previously treated with intravenously administered (IV) glucocorticoids during active TED (overall GO-QOL score $=42.5 \pm 11.7$; includes IV only, oral and IV) had significantly lower overall QOL at the time of survey, during chronic TED, than those who had received only an oral glucocorticoid $(62.7 \pm 17.0, p=0.028)$ or no systemic glucocorticoid $(60.8 \pm 23.1, p=0.042)$.

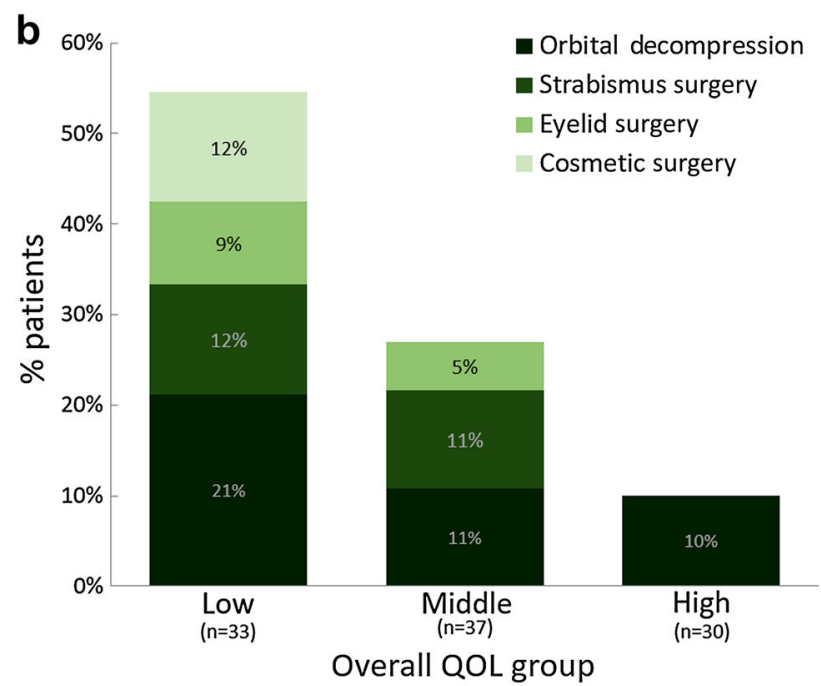

who underwent the listed procedure. It should be noted that some patients in the low and middle QOL groups underwent more than one type of procedure. QOL groups were determined using overall GO-QOL score (low: $\leq 50$ $[n=33]$, middle: between 50 and $75[n=37]$, high: $\geq 75[n=30]) .{ }^{*}$ Indicates statistical significance from the high QOL tercile $(p \leq 0.001)$

\section{DISCUSSION}

This study demonstrates that TED patients in the United States continue to be negatively impacted by their disease years after TED-related inflammation has quiesced. Average TED duration at the time of survey was approximately 6 years, with 3 years of disease inactivity. The most common TED symptom reported during chronic TED was ocular dryness/grittiness, which was not surprising given the direct relationship between TED and dry eye disease [17]. Further, patients with TED have tear film aqueous deficiencies more often than patients with dry eye disease, thought to result from immune-mediated lacrimal gland dysfunction $[17,18]$. QOL remained heavily impaired, as indicated by overall, vision-related, and appearance-related GO-QOL scores that averaged approximately 60 points. Interestingly, these scores were nearly identical to those observed in the teprotumumab clinical trial population of recent-onset ( $\leq 9$ months), 


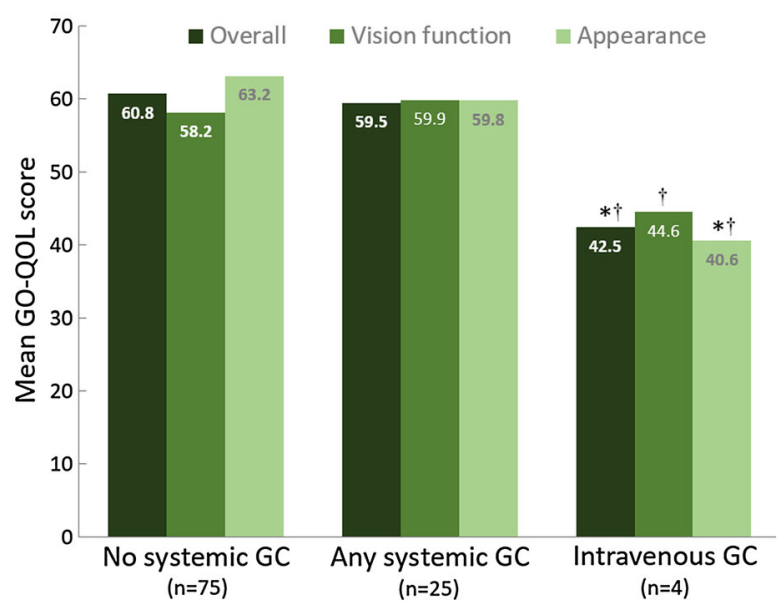

Fig. 3 GO-QOL scores in patients with chronic thyroid eye disease who had and had not been treated with systemic glucocorticoids (GCs) while their TED was in the active phase. ${ }^{*}$ Indicates statistical difference from no systemic GC group $(p \leq 0.04)$. ${ }^{\dagger}$ Indicates statistical difference from patients only administered oral GCs during active TED $(p<0.05)$. GC, glucocorticoid

active, moderate-to-severe TED patients [13]. Our findings are in agreement with patient experiences [19] and prior studies outside of the US, which have shown a marked impact of TED on patient-reported QOL [20-24]. One study of a US population of moderate-to-severe TED patients also demonstrated a decrease in QOL, as reported by physicians [11]. The current study identified several factors that influenced QOL, including the total number of reported TED signs and symptoms. More specifically, pain behind the eyes, blurry vision, light sensitivity, and diplopia were all significantly more prevalent in patients with the lowest QOL. These findings are in agreement with prior studies, which have shown relationships between QOL and TED-related pain [11, 24, 25], vision loss [11], and diplopia [11, 25-29]. The current study also showed that patients who had undergone a surgical procedure for TED had a significantly lower QOL than patients who had not. This finding is in agreement with Terwee et al. [30], who found low mean appearance and visual function GO-QOL scores in chronic TED patients following TED-specific surgical procedures.
The heavy psychosocial health burden of TED has been previously documented in populations around the globe $[8,11,20,21$, $25,26,31-33]$. In agreement, mental health issues were highly prevalent $(42 \%)$ in this cohort of chronic TED patients. This rate was similar to a population of active, moderate-tosevere TED patients (36\%) [11] and was more than twice as frequent as that reported in the general US adult population ("any mental illness" prevalence: $20.6 \%$ [16]). The individual prevalence of anxiety and depression was 34\% and $28 \%$, respectively, nearly identical to a small population of TED patients presenting for orbital decompression surgery (37\% and $26 \%$, respectively [8]). The proportion of patients reporting anxiety increased as QOL decreased (from $17 \%$ in the high QOL group to $48 \%$ in the low QOL group), but rates of depression were not significantly different across QOL groups.

The chronic TED patients included in this study sought medical care for TED approximately 20 times in the year prior to survey. This number increased markedly from approximately 5 TED-related doctor visits/year/patient in the high QOL group to 40 TED-related doctor visits/year/patient in the low QOL group. It is likely that the low QOL group had the highest number of severe TED cases. Prior studies have shown that QOL decreases as TED severity increases [11, 22, 26, 27, 34, 35], and it would be expected that patients with more severe TED would either seek out care more often or be followed more closely by their healthcare provider. However, because our data were patientreported, the clinical severity of TED could not be assessed. A previous report showed that TED patients often have negative interactions with healthcare providers [21] and that quality of care impacts patient QOL [36]. In that study, only one in four patients felt they had been adequately helped to manage the psychological burdens of TED [36]. Further, physician and patient assessments of TED impact often do not align, with physicians tending to underestimate both TED-associated facial changes [37] and QOL impact of medical conditions [38, 39]. All of these factors may have contributed to the increased healthcare utilization by chronic TED patients in the low QOL group. 
Compared to patients who had not received systemic glucocorticoids during active, progressive TED, QOL was the same in patients who had been treated with oral glucocorticoids and significantly higher than in patients who received IV glucocorticoids. However, in the United States, glucocorticoids, particularly IV glucocorticoids, tend to be reserved for the most severe TED patients and those with the most heavily impacted QOL [40]. Therefore, it is likely that QOL was lower in systemic GC-treated patients during acute TED. We also found that the number of surgeries increased as patient QOL decreased. Again, surgery is reserved for patients with more severe TED, and patients undergoing multiple surgeries likely had more severely impacted QOL during acute TED. It should be noted that this survey was completed prior to the commercial availability of teprotumumab, and highlights the extreme unmet need at the time for effective, diseasemodifying treatments for TED.

This study had several limitations. First, our sample size was relatively small. Second, TED phasing and clinical information relied upon patient knowledge of their condition in both the present and the past. Of the 206 patients initially identified as having inactive TED, only 100 were included in analyses. This may have biased our results towards patients who were the least satisfied, because unsatisfied patients tend to be more vocal than satisfied ones. We are confident that patients in this survey had inactive TED, as the vast majority (75\%) of patients reported their physician using at least one confirmatory word/phrase for inactive TED, with $49 \%$ specifically reporting the words "inactive" or "no longer active." However, for patient-reported outcome measures, the Food and Drug Administration (FDA) recommends a short recall period because a patient's current state can influence how they report a past state [41]. Because the survey asked patients to recall information from several years prior, it is possible that recall errors and biases were introduced. That said, the survey results correspond well with what is seen in clinical practice. TED patients with moderate-to-severe disease are often very discontent despite therapeutic interventions, have persistent mental health challenges, and have interpersonal issues that make social and professional relationships difficult. Lastly, our study population was approximately half male, which could be viewed as unexpected given the higher proportion of women reported in the overall TED population [11, 42]. However, this higher female proportion might not be found in more symptomatic chronic, inactive patients, as this has not yet been evaluated in large epidemiological studies. Male and female patients in our study had similar demographics, TED symptom reporting, and overall GO-QOL scores. Therefore, it is unlikely that the high proportion of males influenced study findings for this chronic TED population.

\section{CONCLUSION}

Patient QOL is markedly impacted by TED on a chronic basis, and the QOL scores reported here are very similar to those reported in more active patients. Both patient appearance and visual function remain affected over the long term in many TED patients due to the persistence of TED signs and symptoms into the chronic presentation of the disease. As potential treatments for chronic TED emerge [43, 44], having a better understanding of clinical presentation and impact of QOL becomes increasingly important. Further exploration is warranted to better understand the impact of chronic TED on QOL, particularly with prospective, longitudinal studies that include both patient-reported outcomes and clinical examination.

\section{ACKNOWLEDGEMENTS}

Funding. Sponsorship for this study and the Rapid Service Fee were funded by Horizon Therapeutics plc.

Authorship. All named authors meet the International Committee of Medical Journal Editors (ICMJE) criteria for authorship for this article, take responsibility for the integrity of 
the work as a whole, and have given their approval for this version to be published.

Authorship Contributions. Conceptualization: all authors. Methodology: Noel Stuertz, Lissa Padnick-Silver, Megan-Francis-Sedlak, Robert Holt. Formal analysis and investigation: all authors; Writing - original draft preparation: Lissa Padnick-Silver. Writing - review and editing: all authors. Supervision: Kimberly Cockerham.

Medical Writing, Editorial, and Other Assistance. Medical writing and editing was performed solely by the authors.

Disclosures. This study was funded by Horizon. Dr. Cockerham is on the Horizon Therapeutics Medical Advisory Board, and Lissa Padnick-Silver, Noel Stuertz, Megan FrancisSedlak, and Robert J. Holt are employees of and hold stock in Horizon.

Compliance with Ethics Guidelines. This retrospective study on de-identified patient survey data was reviewed and assigned exempt status by the Western IRB (Puyallup, WA). All study conduct adhered to the tenets of the Declaration of Helsinki.

Data Availability. The datasets generated during and/or analyzed during the current study are owned by the sponsor and are not publicly available.

Prior Presentation. Preliminary data were presented at the 2021 annual meeting of the North American Neuro-Ophthalmology Society (virtual meeting, February 20-23, 2021).

Open Access. This article is licensed under a Creative Commons Attribution-NonCommercial 4.0 International License, which permits any non-commercial use, sharing, adaptation, distribution and reproduction in any medium or format, as long as you give appropriate credit to the original author(s) and the source, provide a link to the Creative Commons licence, and indicate if changes were made. The images or other third party material in this article are included in the article's Creative Commons licence, unless indicated otherwise in a credit line to the material. If material is not included in the article's Creative Commons licence and your intended use is not permitted by statutory regulation or exceeds the permitted use, you will need to obtain permission directly from the copyright holder. To view a copy of this licence, visit http:// creativecommons.org/licenses/by-nc/4.0/.

\section{REFERENCES}

1. Dickinson AJ, Hintschich C. Clinical manifestations. In: Wiersinga WM, Kahaly GJ, editors. Graves' orbitopathy: a multidisciplinary approach-questions and answers. 3rd ed. Basel: Karger; 2017. p. 1-25.

2. Ponto KA, Merkesdal S, Hommel G, Pitz S, Pfeiffer N, Kahaly GJ. Public health relevance of Graves' orbitopathy. J Clin Endocrinol Metab. 2013;98: 145-52.

3. Ponto KA, Pitz S, Pfeiffer N, Hommel G, Weber MM, Kahaly GJ. Quality of life and occupational disability in endocrine orbitopathy. Dtsch Arztebl Int. 2009;106:283-9.

4. Ponto KA, Hommel G, Pitz S, Elflein H, Pfeiffer N, Kahaly GJ. Quality of life in a German Graves orbitopathy population. Am J Ophthalmol. 2011;152:483-90.

5. Terwee C, Wakelkamp I, Tan S, Dekker F, Prummel MF, Wiersinga W. Long-term effects of Graves' ophthalmopathy on health-related quality of life. Eur J Endocrinol. 2002;146:751-7.

6. Jensen AL, Harder I. The impact of bodily change on social behaviour in patients with thyroid-associated ophthalmopathy. Scand J Caring Sci. 2011;25:341-9.

7. Abraham-Nordling $\mathrm{M}$, Törring $\mathrm{O}$, Hamberger $\mathrm{B}$, et al. Graves' disease: a long-term quality-of-life follow up of patients randomized to treatment with antithyroid drugs, radioiodine, or surgery. Thyroid. 2005;15:1279-86.

8. Wickwar S, McBain HB, Ezra DG, Hirani SP, Rose GE, Newman SP. Which factors are associated with quality of life in patients with Graves' orbitopathy presenting for orbital decompression surgery? Eye (Lond). 2015;29:951-7. 
9. Patel P, Khandji J, Kazim M. Recurrent thyroid eye disease. Ophthalmic Plast Reconstr Surg. 2015;31: 445-8.

10. Selva D, Chen C, King G. Late reactivation of thyroid orbitopathy. Clin Exp Ophthalmol. 2004;32: 46-50.

11. Wang Y, Sharma A, Padnick-Silver L, et al. Physician-perceived impact of thyroid eye disease on patient quality of life in the United States. Ophthalmol Ther. 2021;10:75-87.

12. Bartley GB, Fatourechi V, Kadrmas E, et al. Longterm follow-up of Graves ophthalmopathy in an incidence cohort. Ophthalmology. 1996;103: 958-62.

13. Kahaly GJ, Douglas RS, Holt RJ, Sile S, Smith TJ. Teprotumumab for patients with active thyroid eye disease: a pooled data analysis, subgroup analyses, and off-treatment follow-up results from two randomised, double-masked, placebo-controlled, multicentre trials. Lancet Diabetes Endocrinol. 2021;9: 360-72.

14. Dietrich A, Taylor $\mathrm{P}$, White $\mathrm{P}$, et al. Establishing the usefulness of the GO-QOL in a UK hospital-treated population with thyroid eye disease in the CIRTED trial. Psychol Health Med. 2018;23:1341-55.

15. Terwee CB, Gerding MN, Dekker FW, Prummel MF, Wiersinga WM. Development of a disease specific quality of life questionnaire for patients with Graves' ophthalmopathy: the GO-QOL. Br J Ophthalmol. 1998;82:773-9.

16. National Institutes of Health-National Institute of Mental Health. 2021. www.nimh.nih.gov/health/ statistics/mental-illness.shtml. Accessed 4 Jan 2021.

17. Selter JH, Gire AI, Sikder S. The relationship between Graves' ophthalmopathy and dry eye syndrome. Clin Ophthalmol. 2015;9:57-62.

18. Takahashi Y, Lee PAL, Vaidya A, Kono S, Kakizaki H. Tear film break-up patterns in thyroid eye disease. Sci Rep. 2021;11:5288.

19. George J, Patterson N. Graves' Diseae in our own words. Sage Words Publishing: 2010, Webster.

20. Coulter I, Frewin S, Krassas GE, Perros P. Psychological implications of Graves' orbitopathy. Eur J Endocrinol. 2007;157:127-31.

21. Estcourt S, Vaidya B, Quinn A, Shepherd M. The impact of thyroid eye disease upon patients' wellbeing: a qualitative analysis. Clin Endocrinol (Oxf). 2008;68:635-9.
22. Estcourt S, Quinn AG, Vaidya B. Quality of life in thyroid eye disease: impact of quality of care. Eur J Endocrinol. 2011;164:649-55.

23. Park JJ, Sullivan TJ, Mortimer RH, Wagenaar M, Perry-Keene DA. Assessing quality of life in Australian patients with Graves' ophthalmopathy. Br J Ophthalmol. 2004;88:75-8.

24. Gerding MN, Terwee CB, Dekker FW, Koornneef L, Prummel MF, Wiersinga WM. Quality of life in patients with Graves' ophthalmopathy is markedly decreased: measurement by the medical outcomes study instrument. Thyroid. 1997;7:885-9.

25. Kahaly GJ, Petrak F, Hardt J, Pitz S, Egle UT. Psychosocial morbidity of Graves' orbitopathy. Clin Endocrinol (Oxf). 2005;63:395-402.

26. Kahaly GJ, Hardt J, Petrak F, Egle UT. Psychosocial factors in subjects with thyroid-associated ophthalmopathy. Thyroid. 2002;12:237-9.

27. Villagelin D, Romaldini J, Andrade J, et al. Evaluation of quality of life in the Brazilian Graves' disease population: focus on mild and moderate Graves' orbitopathy patients. Front Endocrinol (Lausanne). 2019;10:192.

28. Lin IC, Lee CC, Liao SL. Assessing quality of life in Taiwanese patients with Graves' ophthalmopathy. J Formos Med Assoc. 2015;114:1047-54.

29. Bradley EA, Sloan JA, Novotny PJ, Garrity JA, Woog JJ, West SK. Evaluation of the National Eye Institute visual function questionnaire in Graves' ophthalmopathy. Ophthalmology. 2006;113:1450-4.

30. Terwee CB, Dekker FW, Mourits MP, et al. Interpretation and validity of changes in scores on the Graves' ophthalmopathy quality of life questionnaire (GO-QOL) after different treatments. Clin Endocrinol (Oxf). 2001;54:391-8.

31. Lee H, Roh HS, Yoon JS, Lee SY. Assessment of quality of life and depression in Korean patients with Graves' ophthalmopathy. Korean J Ophthalmol. 2010;24:65-72.

32. Yeatts RP. Quality of life in patients with Graves ophthalmopathy. Trans Am Ophthalmol Soc. 2005;103:368-411.

33. Farid M, Roch-Levecq AC, Levi L, Brody BL, Granet DB, Kikkawa DO. Psychological disturbance in graves ophthalmopathy. Arch Ophthalmol. 2005;123:491-6.

34. Choi YJ, Lim HT, Lee SJ, Lee SY, Yoon JS. Assessing Graves' ophthalmopathy-specific quality of life in Korean patients. Eye (Lond). 2012;26:544-51. 
35. Delfino LC, Zunino A, Sapia V, Croome M, Ilera V, Gauna AT. Related quality of life questionnaire specific to dysthyroid ophthalmopathy evaluated in a population of patients with Graves' disease. Arch Endocrinol Metab. 2017;61:374-81.

36. Estcourt S, Hickey J, Perros P, Dayan C, Vaidya B. The patient experience of services for thyroid eye disease in the United Kingdom: results of a nationwide survey. Eur J Endocrinol. 2009;161: 483-7.

37. Terwee CB, Dekker FW, Bonsel GJ, et al. Facial disfigurement: is it in the eye of the beholder? A study in patients with Graves' ophthalmopathy. Clin Endocrinol (Oxf). 2003;58:192-8.

38. Wilson KA, Dowling AJ, Abdolell M, Tannock IF. Perception of quality of life by patients, partners and treating physicians. Qual Life Res. 2000;9: 1041-52.

39. Ysrraelit MC, Fiol MP, Gaitan MI, Correale J. Quality of life assessment in multiple sclerosis: different perception between patients and neurologists. Front Neurol. 2017;8:729.
40. Wang Y, Sharma A, Padnick-Silver L, et al. Trends in treatment of active, moderate-to-severe thyroid eye disease in the United States. J Endocrine Soc. 2020;4:1-11.

41. US Food and Drug Administration. Patient-reported outcome measures: use in medical product development to support labeling claims: guidance for industry. 2009. https://www.fda.gov/media/77832/ download. Accessed 11 July 2021.

42. Bartley GB, Fatourechi V, Dadrmas EF, et al. The incidence of Graves' ophthalmopathy in Olsted County. Minnesota Am J Ophthalmol. 1995;120: 511-7.

43. Ozzello DJ, Dallalzadeh LO, Liu CY. Teprotumumab for chronic thyroid eye disease. Orbit. 2021 [Epub ahead of print].

44. Ugrader S, Kang J, Lossler AL, et al. Teprotumumab for the treatment of chronic thyroid eye disease. Eye. 2021 [In press]. 